Revue internationale P.M.E.

Économie et gestion de la petite et moyenne entreprise

\title{
La gestion des ressources humaines des entreprises fournisseurs-partenaires : l'impact de la politique d'achat d'un grand groupe donneur d'ordres
}

\section{Florence Laval}

Volume 11, numéro 2-3, 1998

URI : https://id.erudit.org/iderudit/1009044ar

DOI : https://doi.org/10.7202/1009044ar

Aller au sommaire du numéro

Éditeur(s)

Presses de l’Université du Québec

ISSN

0776-5436 (imprimé)

1918-9699 (numérique)

Découvrir la revue

Citer cet article

Laval, F. (1998). La gestion des ressources humaines des entreprises

fournisseurs-partenaires : l'impact de la politique d'achat d'un grand groupe donneur d'ordres. Revue internationale P.M.E., 11(2-3), 75-94.

https://doi.org/10.7202/1009044ar
Résumé de l'article

Cette recherche est une étude descriptive et empirique de l'impact de la politique d'achat du groupe industriel Ford sur la GRH des entreprises fournisseurs-partenaires.

En référence aux modèles théoriques proposés par H. Mahé de Boislandelle pour l'étude de la contingence en GRH, cette recherche vise à analyser l'intégration de la fonction sociale dans une relation d'impartition.

Une enquête a été réalisée auprès d'un échantillon de producteurs de biens intermédiaires, achetés localement par les usines Ford et à l'échelon européen. Il apparaît que le degré de partenariat influence la GRH des entreprises fournisseurs-partenaires, qui revêt de ce fait trois configurations distinctes. Le contenu de ces mix sociaux identifiés respectivement comme une perception contraignante de la GRH, une vision stratégique de cette fonction et une politique sociale stimulée parles exigences en matière d'assurance qualité du donneur d'ordres, est spécifique en termes de pratiques de gestion et d'effets sur l'organisation. 


\title{
La gestion des ressources humaines des entreprises fournisseurs-partenaires: l'impact de la politique d'achat d'un grand groupe donneur d'ordres
}

Florence LAVAL Institut d'administration des entreprises (IAE) de Poitiers

\section{MOTS CLÉS}

\section{Gestion des ressources humaines - Intégration - Partenariat Société Ford - Contingence - Mix social}

\begin{abstract}
RÉSUMÉ
Cette recherche est une étude descriptive et empirique de l'impact de la politique d'achat du groupe industriel Ford sur la GRH des entreprises fournisseurs-partenaires.

En référence aux modèles théoriques proposés par $H$. Mahé de Boislandelle pour l'étude de la contingence en $\mathrm{GRH}$, cette recherche vise à analyser l'intégration de la fonction sociale dans une relation d'impartition.

Une enquête a été réalisée auprès d'un échantillon de producteurs de biens intermédiaires, achetés localement par les usines Ford et à l'échelon européen.

II apparaît que le degré de partenariat influence la GRH des entreprises fournisseurs-partenaires, qui revêt de ce fait trois configurations distinctes. Le contenu de ces mix sociaux identifiés respectivement comme une perception contraignante
\end{abstract}

\section{L'AUTEURE}

Florence LAVAL est actuellement maître de conférences en sciences de gestion à l'Institut d'administration des entreprises (IAE) de Poitiers et membre du CEREGE, équipe "GRH et Performance ". Ses travaux portent sur la GRH des entreprises sous-traitantes. Elle pilote, sous la responsabilité de M. Kalika, une Action Intégrée entre l'IAE et l'Université de Marrakech. Ce programme de recherche financé par le ministère des Affaires étrangères s'intitule : "L'intégration de la GRH dans les relations de partenariat industriel France-Maroc ", dure quatre ans et réunit 12 chercheurs, quatre Français et huit Marocains. Adresse : 20, rue Guillaume VII-Le troubadour, BP 63986022 Poitiers Cedex, France. 
de la GRH, une vision stratégique de cette fonction et une politique sociale stimulée par les exigences en matière d'assurance qualité du donneur d'ordres, est spécifique en termes de pratiques de gestion et d'effets sur l'organisation.

\section{ABSTRACT}

This is an descriptive and empiric study of the Ford purchasing policy impact upon the human resources management of partner suppliers. With reference to the model proposed by $\mathrm{H}$. Mahé de Boislandelle, this research aims at first to analyse the social function integration at partner supplier within an external procurement relationship.

An inquiry was carried out from a sample of Ford partner supplier producing intermediate goods bought by local and European Ford factories.

There is some evidence that supplier's HRM correlates with partnership degree. Three configurations of HRM policy appear: constraining concept, strategic vision, stimulated social policy produced by customer's quality requirements. In each above perception, HRM applications and effects were found to be specific.

\section{RESUMEN}

Esta investigación es un estudio descriptivo y empírico del impacto de la política de compra del grupo industrial Ford sobre la GRH de sus empresas asociadas.

En referencia a los modelos teóricos propuestos por $\mathrm{H}$. Mahé de Boislandelle para el estudio de la contingencia de GRH, esta investigación pretende analizar la integración de la función social en una relación de "impartición".

Resulta de ello que el grado de integración mutua influencia la GRH de las empresas asociadas, que reviste así tres configuraciones distintas. El contenido de estos núcleos - identificados respectivamente como una percepción limitadora de la GRH, una visión estratégica de esta función y una política social estimulada por las exigencias de seguridad - es específico en términos de gestión y de influencia sobre la organización.

\section{Introduction}

Cette recherche a pour origine un appel d'offre du ministère du Travail pour l'étude des comportements d'emploi des sous-traitants en France. Il semble intéressant de se demander s'il n'y a pas une évolution des pratiques de gestion et si l'idée couramment admise que le sous-traitant précarise son personnel pour satisfaire les aléas de la production est toujours justifiée. L'originalité pratique de la démarche réside dans le choix du groupe Ford comme point d'ancrage de la recherche. En effet, le directeur des ressources humaines de l'usine Ford de Bordeaux s'est intéressé à ce sujet et nous a orientée vers les responsables de l'assurance qualité fournisseurs, membres du service européen des achats, afin qu'ils coordonnent cette étude, à titre d'experts. Ainsi, l'étude se consacre à l'impact de la politique d'achat d'un grand groupe industriel sur la gestion des ressources humaines (GRH ) des entreprises fournisseurs-partenaires. 
Partant du constat que les politiques sociales mises en œuvre par les réseaux d'entreprises industrielles sont perçues, à l'heure actuelle, de manière implicite (Weiss, 1994, Paché, 1996), certaines questions demeurent sans réponses. En particulier : la GRH des fournisseurs et des sous-traitants est-elle spécifique ? Ne doit-on pas considérer le donneur d'ordres, par le truchement de ses auditeurs, comme un acteur de changement (Louart, 1991) ? La GRH n'est-elle pas une composante fondamentale de la gestion des entreprises fournisseurs-partenaires ? Donne-t-elle la capacité de s'adapter au changement?

La synthèse de ces interrogations se formule comme suit : Comment s'intègre la GRH d'une entreprise fournisseur-partenaire dans une relation d'impartition ${ }^{1}$ ?

Cette problématique présente l'intérêt d'une modélisation descriptive, éclairante des stratégies relationnelles et de leurs conséquences pour les organisations concernées. C'est également une approche interorganisationnelle du tissu industriel et transversale de l'entreprise.

L'objectif poursuivi est de conduire une recherche empirique finalisée en GRH et appliquée à un contexte industriel particulier. Dans un premier temps, nous proposerons une description des relations client-fournisseur, en mettant en évidence les implications sociales de ces échanges. Dans un second temps, nous aborderons les apports de cette recherche pour les théoriciens et les praticiens.

\section{Modélisation de l'influence d'une logique d'impartition sur la gestion des ressources humaines des fournisseurs-partenaires}

Dans un premier point, nous exposerons notre méthodologie, puis le résultat de l'analyse de phénomènes complexes à la fois transversaux et interorganisationnels.

\subsection{Méthodologie}

La conduite de la recherche est basée sur une démarche logicodéductive empiriquement validée sur un échantillon représentatif. Un état de l'art en sciences de gestion permet d'établir un ancrage théorique. Ainsi, la problématique s'analyse à partir des concepts d'impartition (Barreyre, 1968, 1978, 1992), d'intégration (Lawrence et Lorsch, 1967 ; Håkansson, 1982) et de mix social (Mahé de Boislandelle, 1988). L'impartition explique la répartition des activités en milieu industriel. L'intégration fait état des processus d'interaction interorganisationnels et des modalités de gestion induites. Et le mix social regroupe l'ensemble des domaines abordés par la fonction GRH.

1. Impartir, c'est choisir de faire faire plutôt que de faire soi-même (Barreyre, 1992). 
L'étude des concepts et notamment celle de l'intégration conduit à un cadrage épistémologique. Nous abordons plusieurs phénomènes considérés comme autant de systèmes à l'intérieur et à l'extérieur des frontières du système de la quasi-firme (Eccles, 1981 ; Blois, 1972), c'est-à-dire de l'entreprise intégrée au tissu industriel de la construction automobile. Sous cet angle, on peut identifier

- des variables indépendantes, relatives à la politique d'achat des donneurs d'ordres,

- des variables interdépendantes, liées à la gestion des relations interorganisationnelles, nées de l'impartition,

- des variables dépendantes, constituant le mix social du fournisseur-partenaire.

Nous considérons, de ce fait, la GRH à partir d'un métasystème intégrant ces variables et représentatif des relations industrielles. La fonction GRH est donc analysée à partir d'un système de déterminants comprenant des variables environnementales et organisationnelles dont les combinaisons influencent les pratiques et les effets sociaux. Le choix d'une approche objective et fonctionnaliste semble approprié, compte tenu de la nouveauté et de la richesse du phénomène, mais également de sa complexité. En outre, le modèle sera contingent, de façon à rendre compte de la spécificité et de la diversité des organisations.

À partir de là, les techniques mises en œuvre sont les suivantes :

- réalisation d'une étude exploratoire auprès d'experts et d'acteurs prenant part à la GRH des firmes fournisseurs-partenaires. Son intérêt est d'explorer le problème sur le terrain et de réaliser une étude de la politique d'achat du groupe industriel Ford, préalablement à l'enquête principale. Nous considérons qu'il faut «objectiver» ce facteur de contingence non maîtrisable par le fournisseur ou sous-traitant, car il est conçu a priori (par rapport à la relation d'impartition) par les donneurs d'ordres ;

- réalisation d'une étude empirique descriptive, soit une enquête intensive conduite selon une approche qualitative interprétative par entretien semidirectif en profondeur. Le questionnaire d'enquête est un formulaire de 15 pages comprenant des questions fermées, semi-ouvertes à modalités prédéterminées et ouvertes, centrées. Elles se regroupent en trois parties:

- une étude qualitative : recueil d'informations permettant de caractériser l'entreprise et de connaître les déterminants du système de GRH à partir des déclarations des dirigeants ${ }^{2}$;

2. Directeur général, directeur commercial, directeur de la production, directeur qualité et directeur des ressources humaines. 
- une étude quantitative : recueil d'informations chiffrées inspiré du bilan social pour décrire le système de GRH de la firme et l'évaluer ;

- une évaluation du système qualité du fournisseur selon la procédure d'audit Ford : administration d'un questionnaire et visite d'entreprise (Ford Corporate Quality Office, 1990).

La méthode de sélection de l'échantillon s'appuie sur les facteurs de spécification suivants :

- le secteur d'activité, pour respecter un quota par spécialité industrielle;

- la taille : l'entreprise doit être de taille moyenne, de façon à atténuer l'impact de ce facteur de contingence ;

- l'unité de production qui doit être un centre de décision;

- le stade dans la procédure d'achat, afin que les firmes se trouvent dans des phases différentes du processus ;

- la qualité de la relation interpersonnelle avec la personne sollicitée pour coordonner l'enquête.

Vingt fournisseurs ou sous-traitants ont accepté de participer à l'enquête, soit $20 \%$ de la population mère définie à partir des fichiers du service achat de Ford. Un contrôle rigoureux de la représentativité fut effectué par les responsables du service assurance qualité fournisseur qui supervisèrent l'enquête.

Cette population, dont la taille moyenne est de 378 salariés, se compose de deux familles d'entreprises :

- celle, locale, des fournisseurs d'outillages et des sous-traitants de dépannage, dans le domaine industriel, qui est en relation avec l'établissement de la société Ford, sis à Bordeaux (Production non matérielle);

- celle des fournisseurs de pièces ou d'opérations qui entrent dans la composition du produit fini fabriqué en Europe dans plusieurs usines Ford (Production matérielle).

Ces firmes, établies en France, sont en relation d'affaires avec Ford - relation de fourniture, fourniture spéciale, sous-traitance d'opération ou sous-traitance générale - et bénéficient d'un degré de partenariat très variable, selon la catégorie dans laquelle elles se trouvent et leurs caractéristiques intrinsèques.

Le traitement des données recueillies est un déroulement d'étapes allant du dépouillement des formulaires d'entretien au traitement statistique des données quantitatives dans le but d'opérationaliser les pôles d'un cadre d'analyse et de tester sa cohérence. 
TABLEAU 1

Caractéristiques de l'échantillon d'entreprises fournisseurs ou sous-traitantes

\begin{tabular}{lcc}
\hline Secteur d'activités & Chiffre d'affaires en KF & Effectif \\
\hline Production non matérielle & 60000 & \\
Mécanique & 60000 & 120 \\
Mécanique - électronique & 39000 & 130 \\
Mécanique & 25000 & 69 \\
Mécanique de précision & & 90 \\
Production matérielle & 175000 & \\
Pièces de mécanique en alliages frittés & 160000 & 243 \\
Mécanique & 160000 & 250 \\
Décolletage & 190000 & 409 \\
Coussinets minces & 120000 & 333 \\
Pièces en caoutchouc & 570000 & 280 \\
Fonderie & 231928 & 576 \\
Forge & 71000 & 405 \\
Forge & 650000 & 79 \\
Fonderie & 146000 & 920 \\
Tubes et pièces & 952000 & 206 \\
Tubes et pièces & 260000 & 770 \\
Barres d'acier & 325000 & 590 \\
Pièces en caoutchouc & 350000 & 879 \\
Pièces en caoutchouc & 135000 & 780 \\
Roulements à bille & & 180 \\
Plasturgie & & \\
\hline
\end{tabular}

\subsection{Modélisation de l'intégration de la GRH dans une relation d'impartition}

Les cinq pôles du modèle se caractérisent ainsi :

Statut du fournisseur: il s'agit de connaître la nature de l'entreprise compte tenu de sa dimension (Lawrence et Lorsch, 1967 ; Julien et Marchesnay, 1988), sa nationalité, son propriétaire (ou ses actionnaires) [Marchesnay, 1979; Weiss, 1992] et du type de production (Håkansson et al., 1982; Valla, 1987).

Stratégie relationnelle: la relation de l'entreprise avec son environnement peut être considérée comme un processus qui interagit sur le système de GRH (Mahé de Boislandelle, 1988). La relation est marquée par l'influence du client ou donneur d'ordres, mais également par la stratégie commerciale du fournisseur (Commissariat Général au Plan, 1990 ; Gorgeu et Mathieu, 1990 ; Altersohn, 1992).

Interface organisationnelle : l'organisation du fournisseur s'analyse en termes de structures et de flux. Ce pôle désigne plus précisément les moyens mis en œuvre pour gérer les transactions entre firmes (Valla, 1987; Gorgeu et Mathieu, 1990; 
FIGURE 1

Modèle général de l'intégration du système de GRH dans une relation d'impartition

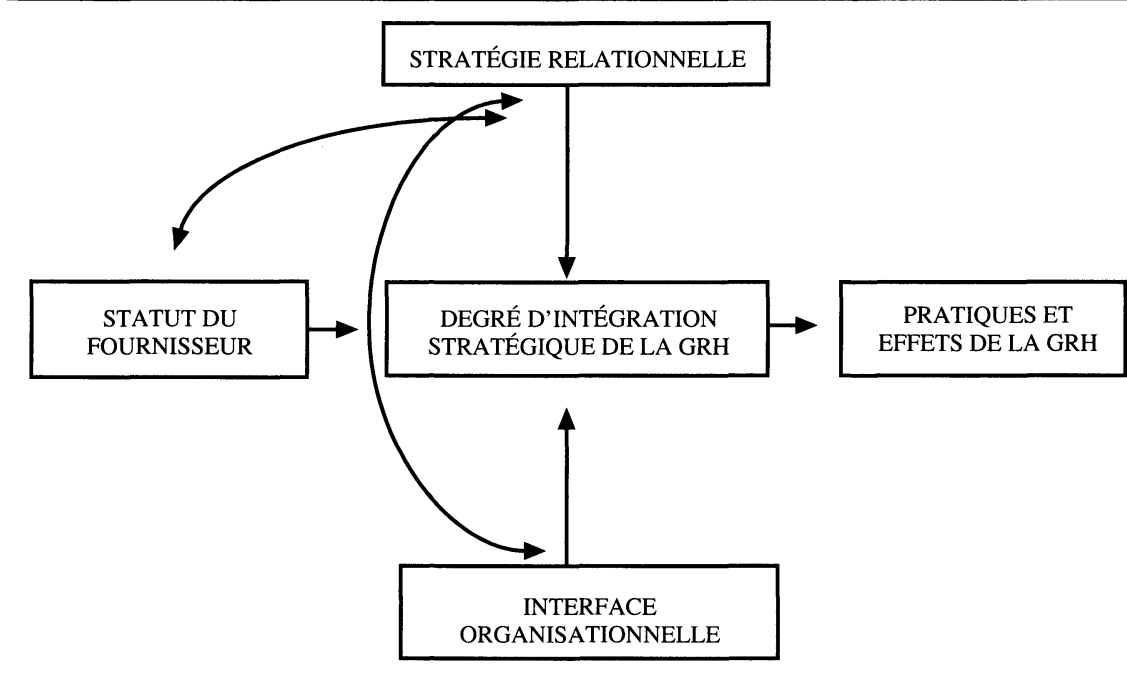

Altersohn, 1992). Concrètement, il s'agit de faire face aux audits, à la mise au point des produits, à la logistique et aux incidents (Commission technique de la soustraitance, 1987 ; Ford Corporate Quality Office, 1990).

Les pratiques de gestion relèvent de différentes fonctions : RD, production, qualité, GRH , et sont plus ou moins coordonnées.

Degré d'intégration stratégique du système de GRH: la conception des $\mathrm{RH}$ dans l'entreprise s'évalue en termes de degré d'intégration stratégique. L'intégration stratégique se définit comme une formulation de la stratégie en considérant systématiquement et de la même manière les aspects $\mathrm{RH}$ et les aspects de positionnement concurrentiel (Lengnick-Hall, 1988 ; Louart, 1991 ; Besseyre des Horts, 1991). Elle résulte du principe de cohérence entre les différentes stratégies fonctionnelles (Barreyre, 1978 ; Schuler et Jackson, 1987 ; Schuler et Walker, 1990).

Pratiques et effets de la GRH : c'est le sous-système de gestion des RH qui se décrit par un ensemble de pratiques dans les domaines de l'emploi, de la rémunération, de la valorisation et de la participation produisant des effets directs (dans les domaines précités), mais aussi des effets indirects, résultant de leur conjugaison spécifique au sein d'une entreprise ou d'un espace organisationnel à un moment donné, sur le plan du climat social, de l'image sociale et des performances technicoéconomiques (Mahé de Boislandelle, 1988). 
Les quatre pôles définis précédemment sont les déterminants de ce sous-système.

L'interface organisationnelle est le pivot de ce modèle de déterminants du système de GRH. Ce pôle décrit les structures mises en œuvre pour gérer la relation avec le client. Ces structures sont contingentes. Elles varient d'une entreprise à l'autre, selon le statut et la stratégie relationnelle affichée. L'observation de l'organisation, en particulier celle des structures et des processus de gestion de la relation interorganisationnelle, révèle le degré d'intégration stratégique du mix social, dont dépendent la nature et l'importance des pratiques et des effets de GRH (Besseyre des Horts, 1987; Bartoli, 1991). Cela revient à étudier la dimension organisationnelle des choix stratégiques (Koenig, 1990) ou, plus précisément, à analyser la cohérence entre l'orientation stratégique et la nature des activités de GRH, considérées comme critiques, dans la mise en œuvre de ces choix (Saporta et Lapassouse-Madrid, 1995). Pour Becker et Gerhart (1996), l'adaptation des pratiques de GRH entre elles et avec les objectifs de l'entreprise (bonne combinaison interne et externe ${ }^{3}$ ) permettrait de soutenir un avantage compétitif. Les auteurs spécialistes de ce domaine de recherche (Gorgeu, Mathieu et Gomel, 1987; Gorgeu et Mathieu, 1990; Gorgeu et Mathieu, 1991; Gorgeu, Mathieu et Gomel, 1992 ; Håkansson et al., 1982, Håkansson, 1989 ; Ring et Van de Ven, 1992, 1994) parviennent à des conclusions identiques. Pour eux, les procédures d'institutionnalisation de la relation impliquent une mobilisation des ressources humaines qui permet de répondre aux impératifs de qualité dictés par le donneur d'ordres. La finalité de ce modèle est de produire une représentation objective et fonctionnelle de la GRH des entreprises fournisseurs ou sous-traitantes. Nous avons réalisé un recueil de données et, en particulier, un recensement des pratiques de GRH et de leurs effets afin de dresser une typologie d'entreprises à partir de ce critère.

\section{Les apports de l'application de notre modèle au réseau de fournisseurs du groupe Ford}

L'application de notre modèle au réseau de fournisseurs du groupe Ford Europe nous permet de faire état, à un moment donné, de la configuration du réseau en dressant une typologie d'entreprises. Ensuite, une analyse qualitative des données démontre la contingence et la spécificité des pratiques de GRH. Enfin, nous discuterons de l'impact de la politique d'achat du groupe Ford sur ces pratiques.

\subsection{Plus le degré de partenariat est élevé, plus la GRH des fournisseurs et des sous-traitants est élaborée pour une typologie d'entreprises}

Les données recueillies ont fait l'objet d'un traitement qualitatif puis quantitatif. Dans un premier temps, une analyse logicosémantique conduit à un classement des

3. «Internal and external fit ». 
individus à l'aide de grilles de lectures, puis à des interprétations. Dans un second temps, les conclusions sont corroborées au moyen d'une analyse factorielle des correspondances.

Voici les trois groupes d'entreprises qui se profilent.

Groupe 1 (25 \% des entreprises) : Quand le degré de partenariat est faible, la GRH est considérée comme une contrainte. Ces entreprises, qui subissent une domination du donneur d'ordres Ford, tendent à être de faible dimension, à avoir une philosophie différente de celle de Ford et à ne pas produire de pièces stratégiques pour Ford. Ces transactions de pièces non intégrées au produit fini ne nécessitent pas d'homologation.

Groupe 2 (45\% des entreprises) : Quand le degré de partenariat est élevé, la GRH est considérée comme stratégique. Ces fournisseurs-partenaires sont de grande dimension, ont une philosophie similaire à celle de Ford et produisent des pièces stratégiques. Ils disposent d'avantages concurrentiels leur conférant un degré de leadership sur le marché.

Groupe 3 (30 \% des entreprises) : Quand le degré de partenariat est moyen, la GRH est considérée comme une politique fonctionnelle parmi d'autres. Ces fournisseurs qui ont une position intermédiaire sont de dimension moyenne et produisent également des pièces essentielles pour Ford. Ils sont en situation d'interdépendance vis-à-vis de Ford et cherchent à évoluer vers le partenariat.

Ce résultat permet de tirer la conclusion suivante : plus le degré de partenariat est élevé, plus la gestion des ressources humaines des fournisseurs ou des soustraitants est élaborée.

La méthode développée par Ford est innovante et témoigne d'une réflexion et de pratiques avancées en matière de partenariat (Altersohn, 1992 ; Gorgeu et Mathieu, 1990). Aussi, notre expérience d'analyse du milieu industriel vécue depuis le service européen des achats du groupe Ford, durant deux ans, paraît significative. En outre, le modèle est construit selon une approche transversale, où l'on considère simultanément les fonctions GRH, achat et qualité, ce qui apporte un nouvel éclairage des phénomènes de mise en réseau (Thorelli, 1987 ; Paché et Paraponaris, 1993).

Ainsi, la représentation théorique proposée et les résultats obtenus soulignent le fait que les systèmes de gestion dépassent les frontières apparentes des organisations et qu'il faut considérer l'impact des relations client-fournisseur dans le cadre d'un métasystème (Pouget et Allard, 1989). Elle démontre que les liens entre firmes, autrement dit, l'intensité ou la qualité du partenariat, ont des effets sur le mix social des entreprises fournisseurs-partenaires. L'intégration de cette fonction relève en partie du degré d'imbrication des échanges et des processus de gestion (Johansson et Mattsson, 1987). Cette imbrication s'analyse à travers les politiques d'achat du groupe industriel. 
Les responsables de la fonction achat d'une entreprise impartitrice telle que Ford apparaissent comme des managers de ressources externes dont l'activité principale est l'audit technique, financier et organisationnel de partenaires privilégiés ou potentiels. La finalité étant de constituer et de promouvoir le réseau de fournisseurs dont ils sont chef de file et de coordonner les activités industrielles afin d'améliorer la compétitivité de cet ensemble (Gouget, Bansard et Salle, 1992; Quelin,1994). Mais toutes les entreprises ne sont pas dans cette situation. Certaines d'entre elles, exclues du panel d'entreprises partenaires, ne sont que fournisseurs ou soustraitantes locales de spécialités ou de produits qui n'entrent pas dans la composition du produit final (l'automobile).

Pour les partenaires, l'audit organisationnel permet aux techniciens de l'assurance qualité fournisseurs du donneur d'ordres de mesurer et de stimuler l'intégration de la fonction sociale à la politique générale du sous-traitant ou fournisseur, au fil de la relation. Il est primordial non seulement de garantir l'équilibre social du réseau, mais également de contribuer à l'amélioration des performances technico-économiques individuelles. Or ces performances représentent un enjeu majeur, sachant qu'elles impliquent la productivité (les coûts) du système de production et la qualité des produits.

\subsection{Le mix social des entreprises fournisseurs et sous-traitantes : des pratiques et des effets de GRH spécifiques.}

Pour appuyer ce premier résultat, nous présentons, ci-après, «le mix social des entreprises sous-traitantes » sous forme de tableaux issus d'une analyse qualitative des données. Le mix social revêt trois configurations distinctes, dont le contenu révèle des pratiques et des effets de GRH spécifiques. L'analyse de contenu des réponses recueillies permet également de définir trois types d'influence qui montrent la nature du lien de la GRH avec le degré de partenariat.

Les trois tableaux ci-après montrent que les entreprises ont parfois une gestion de l'emploi conditionnée par le partenariat. La politique de rémunération n'est pas directement induite par la sous-traitance, sauf en ce qui concerne des primes spécifiques (de chantier). Par contre, la valorisation est un pôle très influencé. Ainsi, un programme de formation au contrôle statistique des procédés (SPC) est mis en place chez les sous-traitants de Ford, car c'est une condition au passage d'ordres. Ford peut également suggérer que le directeur qualité soit associé à l'élaboration du plan de formation. D'une manière générale, l'intégration des fonctions est renforcée à la demande de Ford. Les auditeurs vérifient les procédures, l'affichage dans les ateliers et, plus généralement, la politique d'information et de communication. Les cercles de qualité, autres éléments de gestion participative, dont certains peuvent se déplacer chez le client, sont appréciés et témoignent de l'implication des salariés. 
Nous avons mesuré le climat social et l'image sociale comme variables de résultat et repéré certains dysfonctionnements qui sont, selon le cas, gérés ou ignorés par les firmes. L'évaluation du «SMAC», Supplier Management Awareness \& Commitment, révèle la conception de la GRH , c'est-à-dire l'intérêt porté par les dirigeants à la GRH et à la qualité du management. Toutefois, Ford mesure également les performances technico-économiques (productivité, coût d'obtention de la qualité) de ses fournisseurs.

\section{TABLEAU 2}

\section{Le mix social des entreprises sous-traitantes, soumises à des contraintes (Groupe 1)}

AXE DES POLITIQUES
Gestion de l'emploi

Le travail s'organise par chantier avec des heures supplémentaires, le report des congés et des embauches pour le chantier en CDD ou CTT, ou en stage.

\section{Gestion de la paie}

La rémunération découle de la convention collective de l'UIMM. Des primes de chantiers sont allouées.

\section{Valorisation : respect du cadre légal}

Formation au SPC, en statistiques, au travail sur MCN. Appréciation du personnel et promotions internes. ACT et amélioration des relations hiérarchiques. Enrichissement des postes: définition des fonctions de l'opérateur en matière de contrôle. Délégation et management par objectifs (cadres).

\section{Participation : prise de conscience}

Information du personnel; délégation et dialogue permanent; réunions qualité, affichage de bilans qualité. Quelquefois, cercles de qualité.

\section{RÉSULTATS DES POLITIQUES}

Emploi: précarisé

Le temps de travail est supérieur à la durée légale. On observe une forte mobilité du personnel (embauches et licenciements). Le taux de CDD et de CTT est élevé. La pyramide des âges est souvent déséquilibrée.

Rémunérations faibles

\section{Valorisation}

Le budget de formation atteint l'obligation légale, mais il n'y a pas de politique de valorisation: pas de système d'évaluation du personnel, pas de politique de promotion, pas de politique d'ACT.

\section{Participation}

Pas de politique d'information et de communication: information informelle et procédure d'information administrative. Le nombre d'heures de réunion est faible, il n'y a pas de droit d'expression des salariés. Il n'y a pas de procédures d'accueil. Le nombre de suggestions émises par les salariés est faible.

\section{Climat social passable}

Rotation du personnel (avec départs en cours de période d'essai). Beaucoup de revendications non gérées.

Motivation, adhésion et intégration faibles. Nombre élevé de sanctions. Note de SMAC insuffisante.

\section{Image sociale passable}

D'après le nombre de candidatures spontanées et le faible succès du recrutement, la notoriété interne et externe est faible.

Performances technico-économiques passables

CDD : contrat à durée déterminée; CTT : contrat de travail temporaire ; UIMM : Union des industries mécaniques et métallurgiques ; SPC : contrôle statistique des procédés; $\mathrm{MCN}$ : machine à commande numérique; $\mathrm{ACT}$ : amélioration des conditions de travail; NFOT : nouvelle forme d'organisation du travail ; AMDEC : analyse des modes de défaillance et de leur criticité, méthode d'analyse des défauts potentiels d'un produit ; DOE : design of experiment (plan d'expérience); note de SMAC : élément du questionnaire d'audit utilisé par les auditeurs de Ford, intitulé « supplier awareness and commitment» (prise de conscience et engagement de la direction). 
Lorsque le donneur d'ordres est en position de domination, l'influence s'exerce uniquement sur les pratiques et effets de la GRH. Par exemple, dans le cadre de l'EPA (External Purchasing Assistance ou sous-traitance de capacité), le sous-traitant a recours à une forte précarisation de son personnel. La relation est contraignante dans le sens négatif du terme. Le sous-traitant procède à une gestion administrative des ressources humaines. Cela signifie que les salariés sont essentiellement considérés comme les éléments de fichiers ou de données quantifiées, source de coûts et non de profit (Bartoli, 1991 ; Louart, 1991).

TABLEAU 3

Le mix social des entreprises partenaires (Groupe 2)

\begin{tabular}{l}
\hline AXE DES POLITIQUES \\
Gestion prévisionnelle de l'emploi
\end{tabular}

Mise en œuvre d'un partenariat avec les salariés.

\begin{tabular}{l}
\hline Politique de rémunération et d'implication \\
\hline Équité et transparence du système de rémunération. \\
\hline Stratégie de valorisation \\
\hline
\end{tabular}

Plan de formation pluriannuel : formation à la qualité, formation aux méthodes de gestion de production; accueil et formation des nouveaux embauchés. Évaluation et développement des compétences par un entretien professionnel individualisé. Cours de SPC dispensés au personnel des sous-traitants. Intégration des fonctions qualité et GRH. Analyse de la relation client-fournisseur dans les services administratifs. NFOT, maintenance préventive et contrôle qualité intégré au travail des opérateurs. ACT, hygiène et sécurité.

Stratégie de participation

Programme d'amélioration de l'information et de la communication : aides visuelles, graphiques, diagrammes, procédures écrites explicites, journal d'entreprise, messagerie. Participation par des réunions d'expression des salariés, des "groupes d'implication des salariés », des groupes de travail, des cercles de qualité.

RÉSULTATS DES POLITIQUES

Réduction de la durée du travail et recours au temps partiel. Stabilité des effectifs : peu d'embauches et de rares licenciements. L'effectif permanent représente plus de $95 \%$ de l'effectif total. Pyramide des âges équilibrée.

\begin{tabular}{l}
\hline Rémunérations élevées \\
\hline Valorisation \\
\hline $5 \%$ de la masse salariale est consacré à la formation. \\
L'évaluation des salariés permet une politique de promotion. \\
Il existe une politique d'amélioration des conditions de \\
travail.
\end{tabular}

\section{Participation}

Engagement de moyens pour la communication interne et la participation des salariés. Nombre élevé d'heures de réunions et nombre élevé de suggestions.

Climat social excellent

Faible rotation du personnel (licenciements, démissions). Peu de revendications, gérées. Absentéisme mesuré. Motivation, adhésion, intégration élevées. Nombre de sanctions faibles. Note de SMAC excellente.

Image sociale excellente

Candidatures spontanées et succès du recrutement élevé.

Notoriété interne et externe variable.

Performances technico-économiques excellentes 
Lorsque le degré de partenariat est élevé, l'influence s'exerce essentiellement sur la vision de la GRH. L'entreprise conçoit une gestion stratégique des ressources humaines. Les partenaires échangent des points de vue, se concertent, mais la stratégie est conçue et mise en œuvre par le groupe fournisseur. Il s'agit d'un ensemble de choix explicites définis avec une projection pluriannuelle. Ces pratiques sont cohérentes à long et moyen terme, entre elles et avec les autres choix technicoéconomiques.

\section{TABLEAU 4}

\section{Le mix social des entreprises en voie de partenariat, stimulées par le donneur d'ordres (Groupe 3)}

\begin{tabular}{l} 
AXE DES POLITIQUES \\
\hline Politiques de l'emploi \\
\hline Fidélisation des cadres et licenciement des personnes ne \\
pouvant pas prendre part au développement de l'entreprise. \\
Procédure formalisée de recrutement. Embauche pour \\
renforcer la fonction qualité. \\
\hline Gestion des rémunérations \\
Inspirée de la convention collective de l'UIMM. \\
\hline Politique de valorisation \\
Plan de formation pluriannuel : formation du personnel \\
(cadres et ouvriers) au SPC, à l'AMDEC et au DOE. \\
Certaines formations sont assurées par Ford. \\
Responsabilisation du personnel, enrichissement et \\
élargissement des tâches. Évaluation. \\
\hline Politique de participation
\end{tabular}

Programme d'amélioration de l'information et de la communication : aides visuelles, graphiques, diagrammes, réunions qualité. Participation: cercles de qualité ou réunions d'expression des salariés, cercles SPC, équipes transfonctionnelles.

\begin{tabular}{l} 
RÉSULTATS DES POLITIQUES \\
\hline Emploi : maitrisé \\
\hline La durée du travail est égale à la durée légale. On \\
observe peu de licenciements et de recrutements, très \\
peu de CDD, des CTT et du temps partiel. La pyramide \\
des âges est souvent déséquilibrée \\
\hline Rémunérations moyennes \\
\hline Rémunérations dans la moyenne de la profession \\
\hline Valorisation
\end{tabular}

Le budget de formation atteint $2 \%$ de la masse salariale. Le personnel est évalué. Il y a parfois entretien individuel. Quelques entreprises ont une politique de promotion. Il n'y a pas de politique d'ACT.

\section{Participation}

Bonne utilisation de l'affichage avec des procédures d'information. Le nombre d'heures de réunion est moyen (y compris les réunions d'expression des salariés). Le nombre de suggestions émises par les salariés est moyen.

\section{Bon climat social}

La rotation du personnel s'explique par des démissions et des licenciements. Le nombre de revendications est moyen, elles sont gérées. Absentéisme calculé. Motivation, adhésion et intégration très élevées. Nombre moyen de sanctions. Note de SMAC satisfaisante.

\section{Bonne image sociale}

Candidatures spontanées et succès du recrutement moyen. Notoriété interne et externe convenable.

Bonnes performances technico-économiques

Lorsque le degré de partenariat est moyen, l'influence s'exerce à la fois sur la vision et les pratiques de GRH (y compris la prise en compte de leurs effets). Le donneur d'ordres conseille et assiste le DRH ou le directeur qualité. Des plan d'actions sont conçus et font l'objet d'un suivi. La relation est stimulante dans le 
sens positif du terme. Le fournisseur-partenaire met en œuvre une politique sociale. Le DRH a des responsabilités dans différents domaines (administratif, relations sociales, emploi, formation, etc.) et doit contribuer à la valorisation des potentiels humains par la définition et la mise en œuvre de politiques. Ces politiques visent le développement organisationnel et sont planifiées à moyen terme (Bartoli, 1991).

\subsection{L'impact de la politique d'achat du groupe Ford sur la GRH des fournisseurs : certification et relations interpersonnelles.}

Ce phénomène d'influence explique la spécificité et la contingence de la GRH des entreprises fournisseurs-partenaires. En effet, il montre l'adéquation conjoncturelle et structurelle de la prise en charge de la fonction GRH au processus constructif qu'est la relation d'impartition et en particulier aux démarches d'assurance qualité fournisseur. La conception, les pratiques et les effets de GRH sont «à la hauteur » de la mission de fournisseur de l'industrie (de premier ou de second rang) ou du moins de la perception qu'a l'entreprise de cette mission (Miller, 1985).

Des effets d'apprentissage organisationnels (Ingham, 1994) permettent à une proportion significative d'entreprises de «progresser » dans les divers niveaux de partenariat avec le donneur d'ordres. Or cette progression s'observe simultanément dans les niveaux de formalisation et de sophistication de la GRH.

L'explication réside dans le fait que la relation d'impartition, et particulièrement les procédures d'institutionnalisation de cette relation, stimule l'adoption d'une démarche de gestion systémique des ressources humaines (Louart, 1995):

FIGURE 2

Processus général de régulation des RH

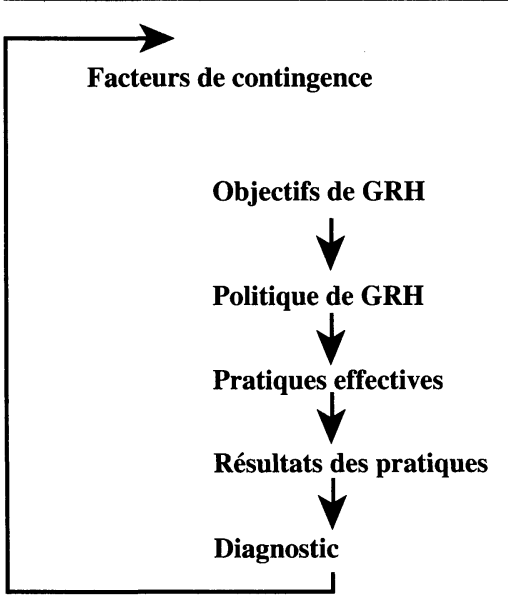

Intention du dirigeant - Conception de la GRH Contexte organisationnel (taille, activité) Environnement légal et institutionnel

Objectifs de GRH

Figure adaptée de Mahé de Boislandelle, 1988.

Choix des indicateurs, évaluation des résultats prise en compte du diagnostic 
Le moteur du système est la recherche de la productivité des membres du réseau et de la compétitivité du produit final (Blois et al., 1990). En effet, les actions (pratiques de GRH) sont suggérées par le donneur d'ordres et les effets organisationnels relèvent de ces actions spécifiques.

L'évaluation des résultats est effectuée de concert avec Ford, ainsi que le diagnostic général du système de gestion, dans le cadre d'audits de conformité. Mais toutes les entreprises ne bénéficient pas des procédures d'assurance qualité fournisseurs. Seules celles des groupes 2 et 3 sont engagées dans le partenariat et ont une communauté d'objectifs avec le donneur d'ordres. Les autres (celles du Groupe 1) sont mises en concurrence, précarisent leur personnel et ne sont pas incitées à modifier leur conception des RH dans le sens du renforcement de l'intégration de ce sous-système de gestion dans le système général de gestion. Il s'agit bien d'une particularité du tissu industriel des firmes produisant des biens intermédiaires qui justifierait la contingence de leur comportement en matière de GRH. Mais ce phénomène explique également l'existence d'une continuité entre les différents états décrits par la typologie (Ring et Van de Ven, 1992, 1994 ; Ingham, 1994). Certaines firmes peuvent «basculer» d'un groupe à l'autre. Par exemple, les entreprises du groupe 3, devenant partenaires du donneur d'ordres, élaborent progressivement une gestion stratégique des ressources humaines. Par contre, l'absence de phénomènes d'apprentissage et de développement organisationnel peut conduire ces mêmes sociétés à rejoindre le groupe 1 . Dans ce cas, la probabilité est forte pour que le résultat des actions mises en œuvre dans le domaine social se dégrade.

Certaines variables du modèle d'analyse ont un caractère déterminant pour l'avenir des fournisseurs et font état du rôle d'agent de changement joué par le donneur d'ordres (Louart, 1991). On peut souligner en premier lieu l'importance des procédures de certification. Leur mise en œuvre par le donneur d'ordres est significative de l'interaction à court terme (homologation), moyen terme (audits de conformité) et long terme (délégation, autonomie). La situation du sous-traitant, dans le sillage de firmes multinationales, possède des avantages à condition de bénéficier d'une démarche d'assurance qualité fournisseurs et d'obtenir une homologation. Cette chance lui donne la possibilité d'harmoniser ses méthodes de gestion avec celles du donneur d'ordres. C'est ainsi que peuvent se transmettre les innovations organisationnelles qui, pour la plupart, sont le fruit de l'expérience et de la réflexion de ces sociétés mondiales. C'est une occasion d'apprentissage et de développement pour les fournisseurs, qu'ils doivent saisir, même au prix d'une perte relative d'autonomie. Ce sentiment est propre à la culture des PME (Marchesnay, 1979 ; Julien et Marchesnay, 1988), mais il devrait être atténué par la volonté de remplir sa mission de fournisseur de premier rang de l'industrie mécanique.

En second lieu, l'importance de la qualité des relations interpersonnelles qui existent entre les auditeurs des donneurs d'ordres et le personnel des sous-traitants chargé de les recevoir est de plus en plus reconnue. Le fait que ces acteurs ne soient 
pas acheteurs réduit les tensions liées à la négociation commerciale. En outre, ces hommes font généralement preuve de compétences en matière de gestion, d'audit et de choix d'actions correctives. L'expérience dont ils se prévalent leur permet d'évaluer très précisément une usine (sa productivité, son potentiel à fabriquer des pièces «bonnes du premier coup»), mais également ses ressources humaines. Ils complètent cet atout par des qualités pédagogiques nécessaires à la prise de conscience des « choses qui ne vont pas » par les dirigeants et à l'enseignement de méthodes et outils de gestion. Une approche moins déterministe mettrait ce phénomène en valeur.

Dès lors que le preneur d'ordres en prendra conscience, il pourra dans un premier temps structurer l'interface organisationnelle et veiller à sa qualité. Pour ce faire, il est souhaitable que les auditeurs aient toujours les mêmes interlocuteurs. Puis, il faut prévoir une méthode d'analyse des rapports d'audit, de compte rendu régulier des résultats destinés au comité de direction qui décidera quelles actions correctives mettre en œuvre et comment les planifier.

\section{Conclusion}

Les conclusions de la recherche s'appliquent à l'ensemble des fournisseurs et partenaires de la société Ford. Elles sont susceptibles d'être généralisées aux fournisseurs et sous-traitants français travaillant pour l'industrie automobile. En effet, les publications du CEE (Centre d'études de l'emploi) [Gorgeu et Mathieu, 1990 ; Gorgeu et Mathieu, 1991 ; Gorgeu, Mathieu et Gomel, 1992] font référence aux pratiques de la société Ford en matière de partenariat et prévoient une évolution des relations interorganisationnelles calquée sur ce modèle dans l'industrie automobile.

Ce phénomène induira une mobilisation des ressources humaines des fournisseurs-partenaires, quel que soit le donneur d'ordres, à condition qu'il adhère à la philosophie du partenariat et mette en œuvre les outils et les moyens nécessaires à sa réalisation (Weiss, 1994).

Par contre, les effets négatifs de la simple mise en concurrence des fournisseurs et des sous-traitants sur l'emploi et les conditions de travail se maintiendront. Ces situations s'observent au sein des unités de production exclues du partenariat par les procédures de certification, en marge des réseaux ainsi constitués.

Il est donc essentiel de retenir l'existence de ces deux niveaux de gestion de la relation client-fournisseur. Le premier est basé sur la confiance, la délégation, l'assistance et la rigueur. Il correspond au partenariat. Le second est plus contraignant, car focalisé sur la réduction du coût d'acquisition des produits. 
Les prolongements envisageables à l'issue de cette recherche pourraient être de deux ordres :

- d'une part, une généralisation de l'étude en intégrant de nouveaux donneurs d'ordres et fournisseurs. Une application élargie du point de vue des individus (l'échantillon doit être plus important) et des variables renforcerait la validité externe de nos résultats. Dans le même ordre d'idée, on peut envisager d'établir une comparaison entre réseaux de nationalités différentes, à l'instar du Commissariat Général au Plan (1990);

- d'autre part, une application similaire aux phénomènes de sous-traitance en cascade constituerait un complément pertinent, eu égard à l'intérêt suscité par cette problématique.

Enfin, les choix méthodologiques pourraient évoluer vers une double approche objective et subjectiviste, afin de prendre en compte les dimensions diachroniques et synchroniques des situations de gestion.

\section{Bibliographie}

ALTERSOHN, C. (1992), De la sous-traitance au partenariat industriel, Paris, L'Harmattan, $302 \mathrm{p}$.

BARREYRE, P.Y. (1968), L'impartition : politique pour une entreprise compétitive, Paris, Hachette.

BARREYRE, P.Y. (1978), «Le choix de la sous-traitance dans la stratégie de l'entreprise », Revue française de gestion, $\mathrm{n}^{\circ} 14$, janvier-février, p. 70-83.

BARREYRE, P.Y. (1992), «La sous-traitance à l'heure des nouvelles politiques d'impartition », Encyclopédie du management, tome 2, coordonnée par J.-P. Helfer et J. Orsoni, Paris, Vuibert, p. 759-772.

BARTOLI, A. (1991), « Stratégie et ressources humaines : analyses cliniques », $2^{\mathrm{e}}$ Congrès de l'AGRH , Cergy, p. 410-419.

BECKER, B. et G. GERHART (1996), « The impact of human resource management on organizational performance : progress and prospects », The Academy of Management, vol. 39, no 4, août, p. 779-801.

BESSEYRE des HORTS, C.H. (1987), «Typologie des pratiques de gestion des ressources humaines », Revue française de gestion, novembre-décembre, p. 149-155.

BESSEYRE des HORTS, C.H. (1991), «L'intégration stratégie-ressources humaines : de la théorie à la pratique », $2^{e}$ Congrès de l'AGRH, Cergy, p. 390-399.

BLOIS, K.J. (1972), «Vertical quasi-integration », Journal of Industrial Economics, juillet, p. 253-272.

BLOIS, K.J. et al., (1990), «Buyer-seller relationships in industrial marketing », Gestion 2000 , vol. $6, \mathrm{n}^{\circ} 3$, p. $73-106$. 
COMMISSARIAT Général au Plan (1990), «Du fordisme au toyotisme ? Les voix de la modernisation du système automobile en France et au Japon », Études et recherches, la Documentation française, $\mathrm{n}^{\text {os }} 7-8$, février, $438 \mathrm{p}$.

COMMISSION TECHNIQUE DE LA SOUS-TRAITANCE (1987), Livre blanc sur le partenariat, éd. AFNOR, Paris, Eyrolles.

ECCLES, R.G. (1981), « The quasifirm in the construction industry », Journal of Economic Behavior and Organization, vol. 2, p. 335-357.

FORD Corporate Quality Office (1990), Guide d'évaluation du système qualité et barèmes, avril, $52 \mathrm{p}$.

FORD Corporate Quality Office (1990), «Classification qualité Q1 pour les fournisseurs de Ford Motor Company », avril, 9 p.

Gorgeu, A. et R. MAthieu (1990), «Partenaire ou sous-traitant ?», Centre d'études de l'emploi, dossier de recherche, $\mathrm{n}^{\circ} 31$, juillet, $93 \mathrm{p}$.

GORGEU, A. et R. MATHIEU (1991), «Les pratiques de livraison en juste-à-temps en France entre fournisseurs et constructeurs automobiles », Centre d'études de l'emploi, dossier de recherche, $\mathrm{n}^{\circ} 41$, décembre, $78 \mathrm{p}$.

GORGEU, A., R. MATHIEU et B. GOMEL (1987), « Les fournisseurs de l'industrie : politique de produit et gestion de la main-d'œuvre », Cahier du centre d'études de l'emploi, $\mathrm{n}^{\mathrm{o}}$ 30, Paris, Presses universitaires de France, p. 31-63.

Gorgeu, A., R. MATHIEU et B. Gomel (1992), «Les nouvelles exigences des donneurs d'ordres : une incitation au dynamisme pour les sous-traitants?», Centre d'études de l'emploi, dossier de recherche, $\mathrm{n}^{\circ} 45,71 \mathrm{p}$.

Gouget, R., D. BANSARD et R. SALLE (1992), «L'établissement des relations fournisseurclient en milieu industriel », Gestion 2000, 6.

HÅKANSSON, H. et al. (1982), International Marketing and Purchasing of Industrial Goods - An Interaction Approach, Toronto, John Wiley and Sons.

HÅKANSSON, H. (1989), Corporate Technological Behavior : Co-operation and Networks, Londres et New York, Routledge.

INGHAM, M. (1994), «L'apprentissage organisationnel dans les coopérations », Revue française de gestion, janvier-février, $\mathrm{n}^{\circ}$ 97, p. 105-121.

JOHANSSON, J. et L.G. MATTSSON (1987), «Interorganizational relations in industrial system : a network approach compared with the transaction-cost approach », International Studies of Management and Organization, vol. 17, $\mathrm{n}^{\circ} 1, \mathrm{p} .34-48$.

JULIEN, P.A. et M. MARCHESNAY (1988), La petite entreprise : principes d'économie et de gestion, Paris, Vuibert-Gestion, $288 \mathrm{p}$.

KOENIG, G. (1990), Management stratégique, vision, manæuvre et tactique, Paris, Nathan, $400 \mathrm{p}$.

LAVAL, F. (1994), La gestion des ressources humaines des entreprises fournisseurspartenaires : l'impact de la politique d'achat d'un grand groupe industriel, Thèse de doctorat en sciences de gestion, Université de Montpellier II, 775 p.

LAWRENCE, P.R. et J.W. LORSCH (1967), Organization and Environment, Boston, Harvard University Press. 
LENGNICK-HALL, C.M. (1988), «Strategic human resources management : a review of the literature and a proposed typology », Academy of Management Review, vol. 13, n 3 , p. 454-470.

LOUART, P. (1991), Gestion des ressources humaines, Paris, Eyrolles, 219 p.

LOUART, P. (1991), «Analyse et optimisation des changements liés aux pratiques de gestion des ressources humaines », $2^{\mathrm{e}}$ Congrès de l'AGRH , Cergy, p. 252-264.

LOUART, P. (1995), Succès de l'intervention en gestion des ressources humaines, Paris, Éditions Liaisons, $314 \mathrm{p}$.

MAHÉ de BoISLANDELLE, H. (1988), Gestion des ressources humaines dans les PME, Paris, Economica, 322 p.

MARCHESNAY, M. (1979), «La dépendance des firmes individuelles : un essai d'analyse et d'application dans leurs relations d'échanges avec les groupes », Économies et sociétés, $\mathrm{n}^{0} 1$, p. 697-733.

MARCHESNAY, M. (1990), «La transaction, outil d'analyse stratégique ?», Cahier de recherche, ERFI, août, $15 \mathrm{p}$.

Miles, R.E. et C.C. SNOW (1984), «Designing strategic human resources system », Organizational Dynamics, vol. 13, n ${ }^{\circ} 1$, p. 36-52.

MiLlER, R. (1985), «Une politique des ressources humaines au service de la stratégie», Revue française de gestion, mars-avril-mai, p. 57-67.

PACHÉ, G. et C. PARAPONARIS (1993), L'entreprise en réseau, Paris, Presses universitaires de France, Collection «Que sais-je ?», 127 p.

PACHÉ, G. (1996), «L'entreprise en réseau entre mythes et réalités », Gestion 2000, 1.

POUGET, M. et F. AllaRd (1989), «Management des ressources humaines et nouvelle démarche industrielle dans un grand groupe industriel: le cas de la gestion de la production », Colloque Management des ressources humaines, Montpellier, $16 \mathrm{p}$.

QuELIN, B. (1994), « Coopération inter-entreprises et création de ressources », Document de travail, Hautes études commerciales, juin, $35 \mathrm{p}$.

RING, P.S. et A.H. VAN DE VEN (1992), « Structuring cooperative relationships between organizations », Strategic Management Journal, vol. 13, p. 483-498.

RING, P. et A.H. VAN DE VEN (1994), «Developmental processes of cooperative interorganizational relationships », Academy of Management Review, vol. 19, $\mathrm{n}^{\circ} 1$, p. $90-118$.

SAPORTA, B. et C. LAPASSOUSE-MADRID (1995), «Les comportements d'intégration marketing-stratégie et leur influence sur la performance de la petite entreprise : un cadre conceptuel préliminaire », Revue internationale $P M E$, vol. 8, $\mathrm{n}^{\circ}$ 2, p. 147-174.

SCHULER, R.S. et S.E. JACKSON (1987), «Linking competitives strategies with human resource management practices », Academy of Management Executive, vol. 1.

SCHULER, R.S. et J.W. WALKER (1990), « Human resources strategy : focusing on issues and actions », Organizational Dynamics, vol. 19.

THORELLI, H. (1987), «Networks : between markets and hierarchies », Strategic Management Journal, vol. $7, \mathrm{n}^{\circ} 1$, p. 37-51. 
VALLA, J.P. (1987), «L'approche interactive. Les travaux du groupe I.M.P. en marketing industriel », Document de travail, IRE Lyon, octobre, $37 \mathrm{p}$.

WEISS, D. (1992), La fonction ressources humaines, Paris, Éditions d'Organisation, 784 p.

WEISS, D. (1994a), «Nouvelles formes d'entreprises et relations de travail », Revue française de gestion, $\mathrm{n}^{\circ} 98$, mars-avril-mai.

WEISS, D. (1994b), «Les nouvelles frontières de l'entreprise », Revue française de gestion, septembre-octobre.

Williamson, O.E. (1973), «Organizational forms and internal efficiency. Markets and hierarchies : some elementary considerations », The American Economic Review, vol. 53, mai, p. 316-325.

WiLliamson, O.E. (1994), Les institutions de l'économie, Paris, InterÉditions. 